\section{Cooled Crystallization Incubators}

The Molecular Dimensions Cooled Crystallization Incubators are specifically selected options from the Series 3000 RUMED Cooled Incubators. This flexible range of incubators, manufactured in Germany, offers remarkable temperature control with minimum vibration and can be customized to suit even the most demanding of laboratory applications. Originally discovered by a leading protein structure laboratory in England RUMED have responded with three superb models for protein crystal growth. Even these base models can be further customized with accessories such as temperature programme control, lighting, glazed door, extra shelves and instrument cable ports.

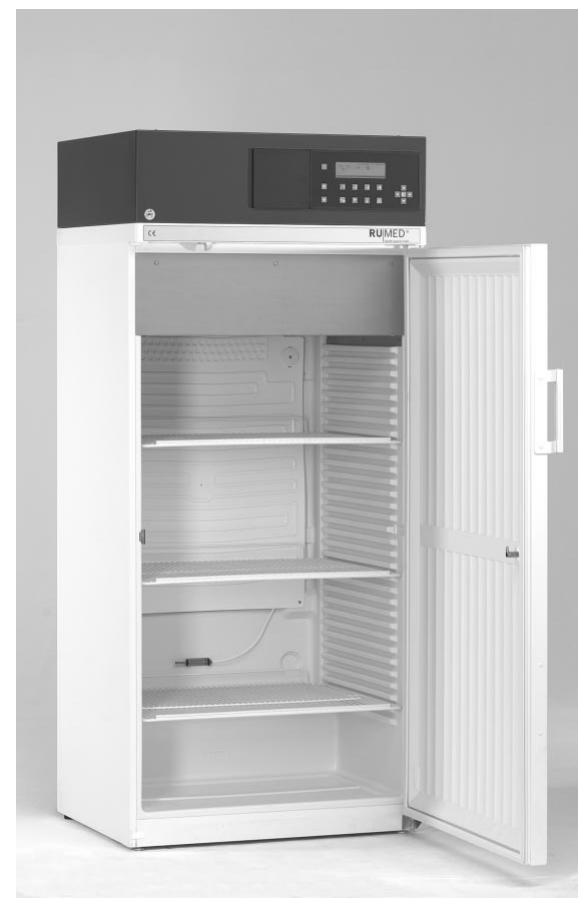

Molecular Dimensions Cooled Incubators from RUMED are a low-cost alternative to building temperature-controlled rooms and provide much more precise control of crystal growth temperature both is respect of time and position of samples. The incubators are specifically designed for crystal growth and feature a white work chamber with plastic coated wire shelves adjustable at $35 \mathrm{~mm}$ intervals. The temperature control is via an electronic controller with digital display, over and under safety cut outs and audible alarms. The doors have a magnetic seal and are fully lockable.

1801,2501 or 3901 models are available. The temperature range is $273 \mathrm{~K}-323 \mathrm{~K}$ with a temperature accuracy of $\pm 273.5 \mathrm{~K}$. They have a well designed control panel and a small floor space footprint.

\section{Molecular Dimensions Ltd}

61-63 Dudley Street

Luton

Bedfordshire

England

\section{New plate reader for DynaPro}

Protein Solutions has developed a 384-well plate reader for its DynaPro light scattering instrument. This new plate reader enables automated, high-throughput dynamic light scattering analysis. The plate reader is based on the company's patented DynaPro dynamic light scattering technology and may either be purchased as a complete system or configured as an add-on to existing DynaPro instruments. DynaPro light scattering instruments were the first commercially available light scattering instruments developed for biomolecular research and especially for pre-screening of protein stock solutions prior to crystallization trials.

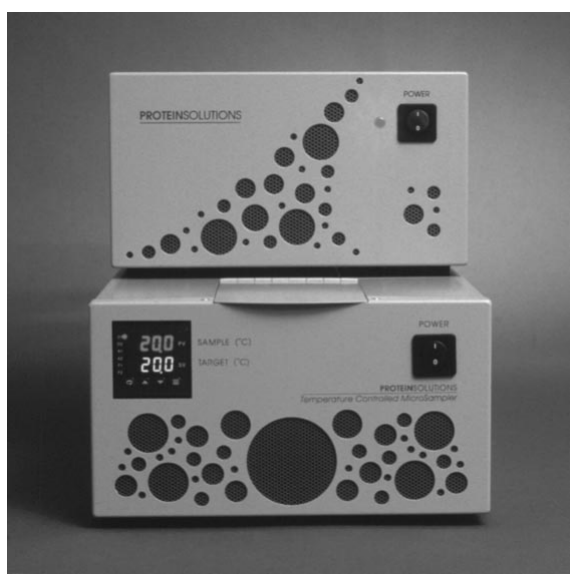

Comprehensive information about protein size, distribution, and aggregation state can be obtained in minutes using as little as $12 \mu \mathrm{l}$ of sample and significantly increase the odds of successful crystallization. Now a high number of samples may be analyzed and scored automatically.

The web address for Protein Solutions is http://www.protein-solutions.com.

\section{Protein Solutions}

750 Vassar Avenue

Lakewood

NJ 08701

USA

\section{New crystallization tool}

Nextal Biotechnologies provides products designed specifically for the experimental needs of crystallization. Nextal's new crystallization tool eliminates the need for grease, facilitates experimetal set-up and crystal recovery, it offers many advantages for screening and optimization of crystallization conditions as well as providing researchers with additional experimental flexibility helping in the improvement of crystallization results.

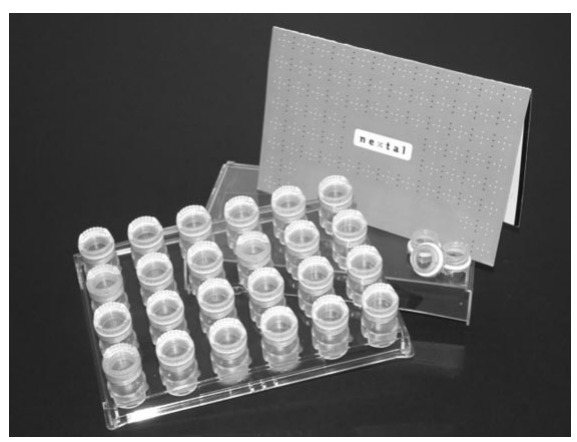

The independently sealed crystallization supports enable users to easily implement experimental strategies like, heavy atom soaking for derivatization of protein crystals, streak seeding, separate nucleation from growth, drug ligand screening and crystallization using the same well.

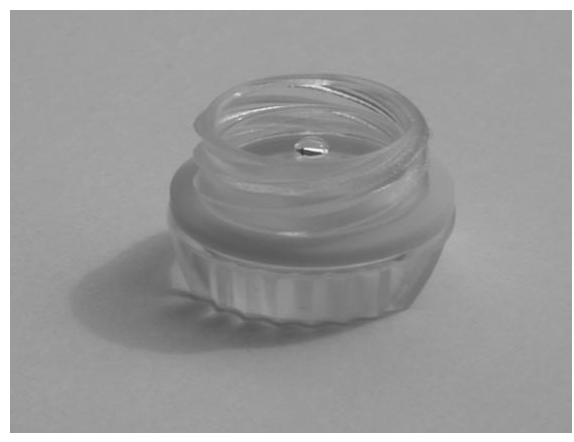

The web address for Nextal is http:// www.nextalbiotech.com.

\section{Nextal Biotechnologies Inc}

1751 Richardson Street
Montreal (Quebec) H3K 1G6
Canada
E-mail: info@nextalbiotech.com 